\title{
End of the road for direct oral anticoagulants in thrombotic APS?
}

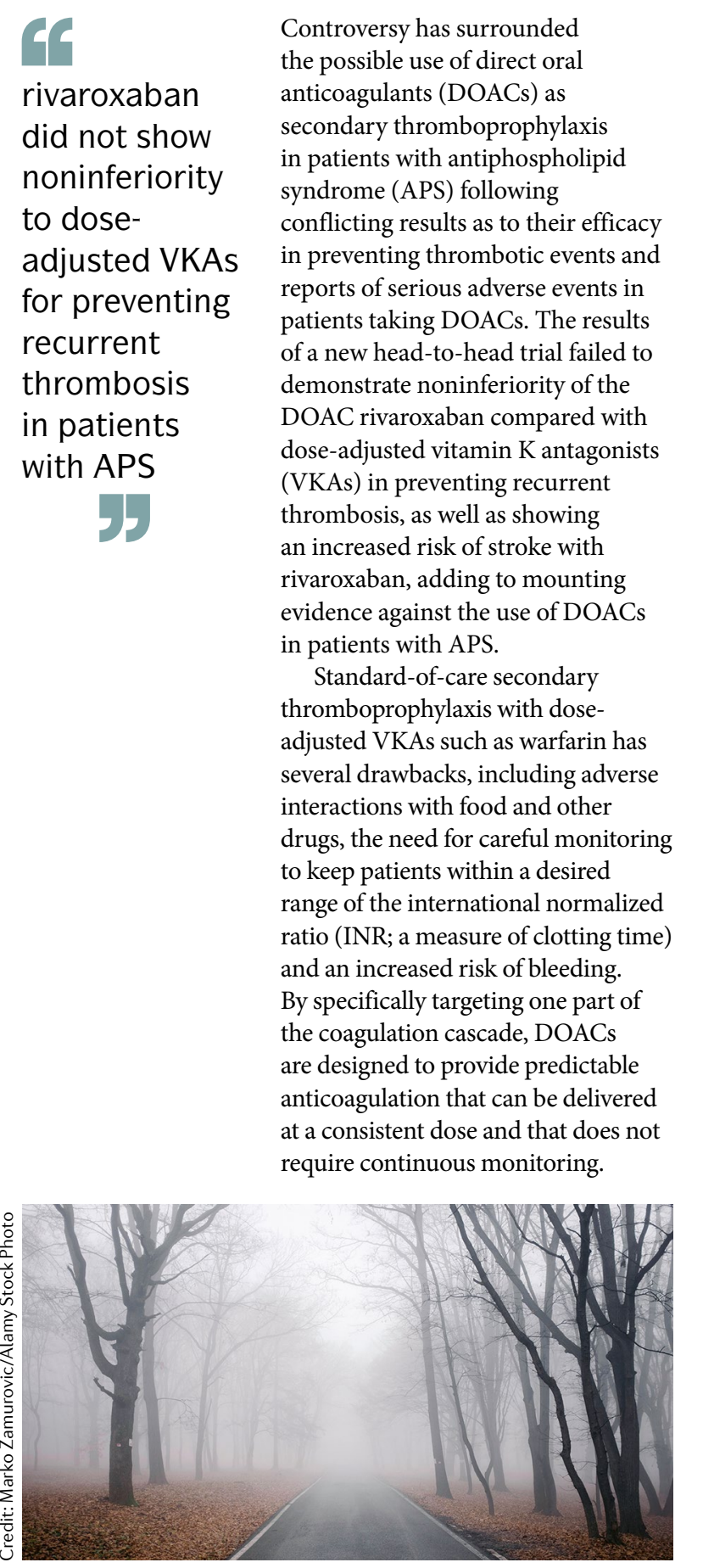

\section{Rivaroxaban directly inhibits} activated factor $\mathrm{X}$ and is approved for use in the prevention of venous thromboembolism in the general population and stroke in patients with acute coronary syndrome, but is not yet approved for use in patients with APS. In the new open-label trial, which was the largest of its kind to date, 190 patients with APS were randomly allocated to receive either $20 \mathrm{mg}$ rivaroxaban per day or VKAs at a dose adjusted to achieve a target INR of 2.0-3.0. The primary efficacy end point was a new thrombotic event occurring during the 36 months of follow-up. Equal numbers of patients receiving each treatment discontinued therapy early for reasons other than thrombotic events, and eight patients died (five of those receiving rivaroxaban and three of those receiving VKAs), mostly as a result of cancer.

In the per protocol analysis, 11 patients receiving rivaroxaban (11.6\%) had a thrombotic event compared with 6 patients receiving VKAs (6.3\%), producing a risk ratio (RR) of 1.83 (CI 0.71-4.76) for rivaroxaban that did not reach statistical significance. The results of the intention-to-treat analysis were similar (RR 2.0; CI 0.78-5.11) and also did not reach statistical significance. The upper limit of the CI margin exceeded the predefined noninferiority margin of 1.40 , leading the authors to conclude that rivaroxaban did not show noninferiority to dose-adjusted VKAs for preventing recurrent thrombosis in patients with APS.

These results contrast with those of the 2016 RAPS trial, which was the first randomized controlled trial to investigate the noninferiority of rivaroxaban compared with warfarin in patients with APS. The threshold for noninferiority was also not met in the RAPS trial, but peak thrombin generation was lower after 6 weeks in those treated with rivaroxaban than in those treated with warfarin, and no thrombotic events were recorded during that time, leading the authors to suggest that rivaroxaban might be considered as an alternative therapy to warfarin in these patients. Differences in the primary efficacy end points and trial durations might explain the discrepancies in results between the current study and the RAPS trial.

Notably, the rate of arterial thrombosis, especially stroke, was much higher in the new study in those receiving rivaroxaban (9 incidences) than in those receiving VKAs (0 incidences). These results echoed those of the 2018 TRAPS study, which examined the noninferiority of rivaroxaban to warfarin for patients with APS who had triple antiphospholipid antibody (aPL) positivity. Although the TRAPS study was terminated early, the results suggested an increased risk of arterial thrombotic events in patients receiving rivaroxaban compared with those receiving warfarin.

The results of the TRAPS study led EULAR to recommend against the use of rivaroxaban in patients with APS who are triple-aPL-positive in their 2019 APS management recommendations. Whether the results of the present trial will lead to this recommendation being extended to all patients with APS remains to be seen.

Joanna Collison

ORIGINAL ARTICLE Ordi-Ros, J. et al. Rivaroxaban versus vitamin $\mathrm{K}$ antagonist in antiphospholipid syndrome: a randomized noninferiority trial. Ann. Intern. Med. https://doi.org/10.7326/M19-0291 (2019) 